\title{
INTUITIONISTIC FUZZY HYPERSOFT SETS
}

\author{
Adem YOLCU ${ }^{1}$, Florentin SMARANDACHE ${ }^{2}$, and Taha Yasin OZTURK ${ }^{1}$ \\ ${ }^{1}$ Department of Mathematics, Kafkas University, 36100, Kars, TURKEY \\ ${ }^{2}$ Department of Mathematics, University of New Mexico, \\ Gallup, NM 87301, USA
}

\begin{abstract}
In this paper, a new environment namely, intuitionistic fuzzy hypersoft set (IFHSS) is defined. We introduce some fundamental operators of intuitionistic fuzzy hypersoft sets such as subset, null set, absolute set, complement, union, intersection, equal set etc. Validity and application are presented with appropriate examples. For greater precision and accuracy, in the future, proposed operations in decision making processes such as personal selection, management issues and others will play a vital role.
\end{abstract}

\section{INTRODUCTION}

The fuzzy set theory identified in 1965 by Zadeh 26 is one of the most popular theories of recent times. Zadeh specified that there is a considerable amount of ambiguity in most real-life situations and physical problems that the classical set theory and its normal mathematical theories centered on such set theory did not give us the necessary knowledge and inferences. Fuzzy set theory has brought a great paradigmatic change in mathematics, but this theory also has some structural difficulties in its nature. Fuzzy set structure is defined with the help of membership function. It's difficult to create a membership function for each event, according to Molodtsov, because creating a membership function is too individual.

Molodtsov 15] introduced the soft set theory in 1999 which he felt was more practical. This theory is a relatively new approach to solving problems involving decision making and uncertainty. The major benefit of soft set theory is that in fuzzy set and other theories it is free from difficulties. Soft set theory has become popular among researchers in a short time and many scientific studies are carried

2020 Mathematics Subject Classification. 03F55, 03E72, 03E75.

Keywords and phrases. Soft set, uncertainty, hypersoft set, intuitionistic fuzzy hypersoft sets . \yolcu.adem@gmail.com-Corresponding author; smarand@unm.edu; taha36100@hotmail.com

(D) 0000-0002-4317-652X; 0000-0002-5560-5926; 0000-0003-2402-6507. 
out on this theory every year 8, 16, 17, 27. Maji et al. 12, 14 proposed an implementation of soft sets focused on parameter reduction to preserve optimum selection artifacts in decision-making problems. Chen 5] investigated a new definition and various applications of the reduction of soft sets to parameters. Pei and Miao 19 have shown that soft sets are a special class of information systems. Kong et al. 9 introduced the reduction and algorithm of soft sets to normal parameters. Zou and Xiao 28 discussed the soft data analysis approach. Aktaş and Çağman 22 defined the algebraic structure of soft set theory.

The intuitionistic fuzzy set (IFS) theory [3,4] was created by adding a nonmembership function to the fuzzy set structure. The non-member function makes IFS more functional in decision-making problems. Maji et al. 10 13] combined soft set theory with the theory of the intuitionistic fuzzy sets and called intuitionistic fuzzy soft Set (IFSS). The parameterization and hesitancy acquired by IFSS from this mixture facilitates very accurately the description of real-world situations. IFSS is a valuable method for addressing data uncertainty and vagueness. Many scientific paper have shown the suitability of IFSS to issue decision making [7,11].

Smarandache [21] introduced a new technique to deal with uncertainty. By converting the function into multiple decision functions, he generalized the soft to hypersoft set. Many studies have been done recently using hypersoft set structure 1. $6,18,20,22,23,24,25]$.

Multi-criteria decision-making $(\mathrm{MCDM})$ is concerned with coordinating and taking care of matters of preference and preparation, including multi-criteria. Intuitionistic fuzzy soft set environments can not be used to solve certain types of problems if attributes are more than one and further bifurcated. Therefore, there was a serious need to identify a new approach to solve such problems, so a new setting, namely the intuitionistic fuzzy hypersoft Sets (IFHSS), is established for this reason. In the present paper, we introduce intuitionistic fuzzy hypersoft set theory. Intuitionistic fuzzy hypersoft set theory is a mixture of IFS theory and the hypersoft set theory. The complement, subset, equal set, "AND", "OR", intersection, union notions are defined on intuitionistic fuzzy hypersoft sets. This paper also is supported by many suitable examples.

\section{Preliminary}

Definition 1. [3] An intuitionistic fuzzy set $H$ in $U$ is $H=\left\{\left(u, \theta_{H}(u), \sigma_{H}(u)\right)\right.$ : $u \in U\}$, where $\theta_{H}: U \rightarrow[0,1], \sigma_{H}: U \rightarrow[0,1]$ with the condition $0 \leq \theta_{H}(u)+$ $\sigma_{H}(u) \leq 1, \forall u \in U . \theta_{H}, \sigma_{H} \in[0,1]$ denote the degree of membership and nonmembership of $u$ to $H$, respectively. The set of all intuitionistic fuzzy sets over $U$ will be denoted by $\operatorname{IFP}(U)$.

Definition 2. [15] Let $U$ be an initial universe and $E$ be a set of parameters. A pair $(H, E)$ is called a soft set over $U$, where $H$ is a mapping $H: E \rightarrow \mathcal{P}(U)$. In other words, the soft set is a parameterized family of subsets of the set $U$. 
Definition 3. [13] Let $U$ be an initial universe and $E$ be a set of parameters. A pair $(H, E)$ is called an intuitionistic fuzzy soft set over $U$, where $H$ is a mapping given by, $H: E \rightarrow \operatorname{IFP}(U)$.

In general, for every $e \in E, H(e)$ is an intuitionistic fuzzy set of $U$ and it is called intuitionistic fuzzy value set of parameter $e$. Clearly, $H(e)$ can be written as a intuitionistic fuzzy set such that $H(e)=\left\{\left(u, \theta_{H}(u), \sigma_{H}(u)\right): u \in U\right\}$.

Definition 4. [21] Let $U$ be the universal set and $P(U)$ be the power set of $U$. Consider $e_{1}, e_{2}, e_{3}, \ldots, e_{n}$ for $n \geq 1$, be $n$ well-defined attributes, whose corresponding attribute values are resspectively the sets $E_{1}, E_{2}, \ldots, E_{n}$ with $E_{i} \cap E_{j}=\emptyset$, for $i \neq j$ and $i, j \in\{1,2, \ldots, n\}$, then the pair $\left(H, E_{1} \times E_{2} \times \ldots \times E_{n}\right)$ is said to be Hypersoft set over $U$ where $H: E_{1} \times E_{2} \times \ldots \times E_{n} \rightarrow P(U)$.

\section{Intuitionistic Fuzzy Hypersoft Sets}

Definition 5. Let $U$ be the universal set and $\operatorname{IFP}(U)$ be the intuitionistic fuzzy power set of $U$. Consider $e_{1}, e_{2}, e_{3}, \ldots, e_{n}$ for $n \geq 1$, be $n$ well-defined attributes, whose corresponding attribute values are respectively the sets $E_{1}, E_{2}, \ldots, E_{n}$ with $E_{i} \cap E_{j}=\emptyset$, for $i \neq j$ and $i, j \in\{1,2, \ldots, n\}$. Let $A_{i}$ be the nonempty subset of $E_{i}$ for each $i=1,2, \ldots, n$. An intuitionistic fuzzy hypersoft set defined as the pair $\left(H, A_{1} \times A_{2} \times \ldots \times A_{n}\right)$ where; $H: A_{1} \times A_{2} \times \ldots \times A_{n} \rightarrow I F P(U)$ and

$H\left(A_{1} \times A_{2} \times \ldots \times A_{n}\right)=\left\{<\xi,\left(\frac{u}{\theta_{H(\xi)}(u), \sigma_{H(\xi)}(u)}\right)>: u \in U, \xi \in A_{1} \times A_{2} \times \ldots \times A_{n} \subseteq E_{1} \times E_{2} \times \ldots \times E_{n}\right\}$

where $\theta$ and $\sigma$ are the membership and non-membership value, respectively such that $0 \leq \theta_{H(\xi)}(u)+\sigma_{H(\xi)}(u) \leq 1$ and $\theta_{H(\xi)}(u), \sigma_{H(\xi)}(u) \in[0,1]$. For sake of simplicity, we write the symbols $\Delta$ for $E_{1} \times E_{2} \times \ldots \times E_{n}, \Omega$ for $A_{1} \times A_{2} \times \ldots \times A_{n}$ and $\xi$ for an element of the set $\Omega$.

Definition 6. i) An intutionistic fuzzy hypersoft set $(H, \Delta)$ over the universe $U$ is said to be null intuitionistic fuzzy hypersoft set and denoted by $0_{\left(U_{I F H}, \Delta\right)}$ if for all $u \in U$ and $\xi \in \Delta, \theta_{H(\xi)}(u)=0$ and $\sigma_{H(\xi)}(u)=1$.

ii) An intutionistic fuzzy hypersoft set $(H, \Delta)$ over the universe $U$ is said to be absolute intuitionistic fuzzy hypersoft set and denoted by $1_{\left(U_{I F H}, \Delta\right)}$ if for all $u \in U$ and $\xi \in \Delta, \theta_{H(\xi)}(\alpha)=1$ and $\sigma_{H(\xi)}(u)=0$.

Example 7. Let $U$ be the set of cars given as $U=\left\{u_{1}, u_{2}, u_{3}\right\}$ also consider the set of attributes as $E_{1}=$ Fuel, $E_{2}=$ Transmission, $E_{3}=$ Color and their respective attributes are given as

$$
\begin{aligned}
& E_{1}=\text { Fuels }=\left\{\operatorname{Gasoline}\left(\alpha_{1}\right), \operatorname{Diesel}\left(\alpha_{2}\right), \operatorname{Electric}\left(\alpha_{3}\right)\right\} \\
& E_{2}=\operatorname{Transmissions}=\left\{\operatorname{Automatic}\left(\beta_{1}\right), \operatorname{Manual}\left(\beta_{2}\right)\right\} \\
& E_{3}=\operatorname{Colors}=\left\{\operatorname{Black}\left(\gamma_{1}\right), \operatorname{Blue}\left(\gamma_{2}\right), \operatorname{White}\left(\gamma_{3}\right)\right\}
\end{aligned}
$$

Suppose that

$$
A_{1}=\left\{\alpha_{3}\right\}, A_{2}=\left\{\beta_{1}, \beta_{2}\right\}, A_{3}=\left\{\gamma_{1}, \gamma_{3}\right\}
$$




$$
B_{1}=\left\{\alpha_{1}, \alpha_{3}\right\}, B_{2}=\left\{\beta_{1}\right\}, B_{3}=\left\{\gamma_{1}, \gamma_{2}\right\}
$$

are subset of $E_{i}$ for each $i=1,2,3$. Then the intuitionistic fuzzy hypersofts $\left(H, \Omega_{1}\right)$ and $\left(G, \Omega_{2}\right)$ defined as follows;

$$
\begin{aligned}
& \left(H, \Omega_{1}\right)=\left\{\begin{array}{c}
<\left(\alpha_{3}, \beta_{1}, \gamma_{1}\right),\left\{\frac{u_{1}}{(0.6,0,3)}, \frac{u_{2}}{(0.4,0.2}\right\}>,<\left(\alpha_{3}, \beta_{1}, \gamma_{3}\right),\left\{\frac{u_{2}}{(0.2,0.6)}, \frac{u_{3}}{(0.3,0.1)}\right\}>, \\
\left.<\left(\alpha_{3}, \beta_{2}, \gamma_{1}\right),\left\{\frac{u_{1}}{(0.7,0.3)}, \frac{u_{2}}{(0.3,0.5)}, \frac{u_{3}}{(0.1,0.6)}\right\}>,<\left(\alpha_{3}, \beta_{2}, \gamma_{3}\right),\left\{\frac{u_{1}}{(0.8,0.2)}, \frac{u_{2}}{(0.2,0.4)}\right\}>\right\rangle
\end{array}\right\} \\
& \left(G, \Omega_{2}\right)=\left\{\begin{array}{c}
<\left(\alpha_{1}, \beta_{1}, \gamma_{1}\right),\left\{\frac{u_{1}}{(0.3,0,3)}, \frac{u_{2}}{(0.1,0.2)}, \frac{u_{3}}{(0.6,0.4)}\right\}>,<\left(\alpha_{1}, \beta_{1}, \gamma_{2}\right),\left\{\frac{u_{1}}{(0.7,0.2)}, \frac{u_{2}}{(0.6,0.1)}\right\}>, \\
<\left(\alpha_{3}, \beta_{1}, \gamma_{1}\right),\left\{\frac{u_{1}}{(0.9,0.1)}, \frac{u_{2}}{(0.2,0.7)}\right\}>,<\left(\alpha_{3}, \beta_{1}, \gamma_{2}\right),\left\{\frac{u_{2}}{(0.5,0.3)}, \frac{u_{3}}{(0.3,0.7)}\right\}>
\end{array}\right\}
\end{aligned}
$$

Tabular form of these sets are given as follows:

TABle 1. Tabular form of IFHSS $\left(H, \Omega_{1}\right)$

\begin{tabular}{|cccc|}
\hline$\left(H, \Omega_{1}\right)$ & $u_{1}$ & $u_{2}$ & $u_{3}$ \\
$\left(\alpha_{3}, \beta_{1}, \gamma_{1}\right)$ & $(0.6,0.3)$ & $(0.4,0.2)$ & $(0,1)$ \\
$\left(\alpha_{3}, \beta_{1}, \gamma_{3}\right)$ & $(0,1)$ & $(0.2,0.6)$ & $(0.3,0.1)$ \\
$\left(\alpha_{3}, \beta_{2}, \gamma_{1}\right)$ & $(0.7,0.3)$ & $(0.3,0.5)$ & $(0.1,0.6)$ \\
$\left(\alpha_{3}, \beta_{2}, \gamma_{3}\right)$ & $(0.8,0.2)$ & $(0.2,0.4)$ & $(0,1)$ \\
\hline
\end{tabular}

TABle 2. Tabular form of IFHSS $\left(G, \Omega_{2}\right)$

\begin{tabular}{|cccc|}
\hline$\left(G, \Omega_{2}\right)$ & $u_{1}$ & $u_{2}$ & $u_{3}$ \\
$\left(\alpha_{1}, \beta_{1}, \gamma_{1}\right)$ & $(0.3,0.3)$ & $(0.1,0.2)$ & $(0.6,0.4)$ \\
$\left(\alpha_{1}, \beta_{1}, \gamma_{2}\right)$ & $(0.7,0.2)$ & $(0.6,0.1)$ & $(0,1)$ \\
$\left(\alpha_{3}, \beta_{1}, \gamma_{1}\right)$ & $(0.9,0.1)$ & $(0.2,0.7)$ & $(0,1)$ \\
$\left(\alpha_{3}, \beta_{1}, \gamma_{2}\right)$ & $(0,1)$ & $(0.5,0.3)$ & $(0.3,0.7)$ \\
\hline
\end{tabular}

Corollary 8. It is clear that each intuitionistic fuzzy hypersoft set is also intuitionistic fuzzy soft set. An example of this situation is provided below.

Example 9. We consider that Example 7. If we select the parameters from a single attribute set such as $E_{1}$ while creating the intuitionistic fuzzy hypersoft set, then the resulting set becomes the intuitionistic fuzzy soft set. Therefore, it is clear that each intuitionistic fuzzy hypersoft set is also intuitionistic fuzzy soft set.That is, the intuitionistic fuzzy hypersoft set structure is the generalized version of the intuitionistic fuzzy soft sets.

Definition 10. Let $U$ be an initial universe set and $\left(H, \Omega_{1}\right),\left(G, \Omega_{2}\right)$ be two intuitionistic fuzzy hypersoft sets over the universe $U$. We say that $\left(H, \Omega_{1}\right)$ is an intuitionistic fuzzy hypersoft subset of $\left(G, \Omega_{2}\right)$ and denote $\left(H, \Omega_{1}\right) \widetilde{\subseteq}\left(G, \Omega_{2}\right)$ if

i) $\Omega_{1} \subseteq \Omega_{2}$

ii) For any $\xi \in \Omega_{1}, H(\xi) \subseteq G(\xi)$,

That is for all $u \in U$ and $\xi \in \Omega_{1}, \theta_{H(\xi)}(u) \leq \theta_{G(\xi)}(u)$ and $\sigma_{H(\xi)}(u) \geq \sigma_{G(\xi)}(u)$. 
Example 11. We consider that attributes in Example 7 and Let $\left(H, \Omega_{1}\right),\left(G, \Omega_{2}\right)$ be two intuitionistic fuzzy hypersoft set over the same universe $U=\left\{u_{1}, u_{2}, u_{3}\right\}$. Tabular forms of $\left(H, \Omega_{1}\right)$ and $\left(G, \Omega_{2}\right)$ are following:

TABLE 3. Tabular form of IFHSS $\left(H, \Omega_{1}\right)$

\begin{tabular}{|cccc|}
\hline$\left(H, \Omega_{1}\right)$ & $u_{1}$ & $u_{2}$ & $u_{3}$ \\
$\left(\alpha_{3}, \beta_{1}, \gamma_{1}\right)$ & $(0.5,0.4)$ & $(0.1,0.8)$ & $(0.3,0.4)$ \\
$\left(\alpha_{3}, \beta_{1}, \gamma_{3}\right)$ & $(0.2,0.7)$ & $(0.3,0.6)$ & $(0.1,0.6)$ \\
$\left(\alpha_{3}, \beta_{2}, \gamma_{1}\right)$ & $(0,1)$ & $(0,1)$ & $(0,1)$ \\
$\left(\alpha_{3}, \beta_{2}, \gamma_{3}\right)$ & $(0,1)$ & $(0,1)$ & $(0,1)$ \\
\hline
\end{tabular}

TABLE 4. Tabular form of IFHSS $\left(G, \Omega_{2}\right)$

\begin{tabular}{|cccc|}
\hline$\left(G, \Omega_{2}\right)$ & $u_{1}$ & $u_{2}$ & $u_{3}$ \\
$\left(\alpha_{3}, \beta_{1}, \gamma_{1}\right)$ & $(0.6,0.1)$ & $(0.2,0.5)$ & $(0.7,0.3)$ \\
$\left(\alpha_{3}, \beta_{1}, \gamma_{3}\right)$ & $(0.6,0.4)$ & $(0.4,0.6)$ & $(0.7,0.4)$ \\
$\left(\alpha_{3}, \beta_{2}, \gamma_{1}\right)$ & $(0,1)$ & $(0,1)$ & $(0,1)$ \\
$\left(\alpha_{3}, \beta_{2}, \gamma_{3}\right)$ & $(0.4,0.3)$ & $(0.8,0.2)$ & $(0.6,0.4)$ \\
\hline
\end{tabular}

It is clear that $\left(H, \Omega_{1}\right) \widetilde{\subseteq}\left(G, \Omega_{2}\right)$. we can also written as

$$
\begin{array}{r}
\left(H, \Omega_{1}\right) \widetilde{\subseteq}\left(G, \Omega_{2}\right)=\left\{\begin{array}{c}
<\left(\alpha_{3}, \beta_{1}, \gamma_{1}\right),\left\{\frac{u_{1}}{(0.5,0,4)}, \frac{u_{2}}{(0.1,0.8)}, \frac{u_{3}}{(0.3,0.4)}\right\}>, \\
<\left(\alpha_{3}, \beta_{1}, \gamma_{3}\right),\left\{\frac{u_{1}}{(0.2,0,7)}, \frac{u_{2}}{(0.3,0.6)}, \frac{u_{3}}{(0.1,0.6)}\right\}>, \\
<\left(\alpha_{3}, \beta_{2}, \gamma_{1}\right),\left\{\frac{u_{1}}{(0,1)}, \frac{u_{2}}{(0,1)}, \frac{u_{3}}{(0,1)}\right\}>, \\
<\left(\alpha_{3}, \beta_{2}, \gamma_{3}\right),\left\{\frac{u_{1}}{(0,1)}, \frac{u_{2}}{(0,1)}, \frac{u_{3}}{(0,1)}\right\}>
\end{array}\right\} \\
\widetilde{\subseteq}\left\{\begin{array}{c}
<\left(\alpha_{3}, \beta_{1}, \gamma_{1}\right),\left\{\frac{u_{1}}{(0,6,1)}, \frac{u_{2}}{(0.2,0.5)}, \frac{u_{3}}{(0.7,0.3)}\right\}>, \\
<\left(\alpha_{3}, \beta_{1}, \gamma_{3}\right),\left\{\frac{\left.u_{1}, 1\right)}{(0.6,0,4)}, \frac{u_{2}}{(0.4,0.6)}, \frac{u_{3}}{(0.7,0.4)}\right\}>, \\
<\left(\alpha_{3}, \beta_{2}, \gamma_{1}\right),\left\{\frac{u_{1}}{(0,1)}, \frac{u_{2}}{(0,1)}, \frac{u_{3}}{(0,1)}\right\}, \\
<\left(\alpha_{3}, \beta_{2}, \gamma_{3}\right),\left\{\frac{u_{1}, 1}{(0.4,0.3)}, \frac{u_{2}}{(0.8,0.2)}, \frac{u_{3}}{(0.6,0.4)}\right\}>
\end{array}\right\}
\end{array}
$$

Definition 12. Let $U$ be an initial universe set and $\left(H, \Omega_{1}\right),\left(G, \Omega_{2}\right)$ be two intuitionistic fuzzy hypersoft sets over the universe $U$. We say that $\left(H, \Omega_{1}\right)$ is an intuitionistic fuzzy hypersoft equal $\left(G, \Omega_{2}\right)$ and denote $\left(H, \Omega_{1}\right) \cong\left(G, \Omega_{2}\right)$ if for all $u \in U$ and $\xi \in \Delta, \theta_{H(\xi)}(u)=\theta_{G(\xi)}(u)$ and $\sigma_{H(\xi)}(u)=\sigma_{G(\xi)}(u)$.

Theorem 13. Let $U$ be an initial universe set, $\Omega_{1}, \Omega_{2}, \Omega_{3} \subseteq \Delta$ and $\left(H, \Omega_{1}\right),\left(G, \Omega_{2}\right)$, $\left(K, \Omega_{3}\right)$ be intuitionistic fuzzy hypersoft sets over the universe $U$. Then,

$$
\begin{aligned}
& \text { i) }\left(H, \Omega_{1}\right) \widetilde{\widetilde{\subseteq}} 1_{\left(U_{I F H}, \Delta\right)} \text {, } \\
& \text { ii) } 0_{\left(U_{I F H}, \Delta\right)} \widetilde{\subseteq}\left(H, \Omega_{1}\right) \text {, } \\
& \text { iii) }\left(H, \Omega_{1}\right) \widetilde{\subseteq}\left(G, \Omega_{2}\right) \text { and }\left(G, \Omega_{2}\right) \widetilde{\subseteq}\left(K, \Omega_{3}\right) \Rightarrow\left(H, \Omega_{1}\right) \widetilde{\subseteq}\left(K, \Omega_{3}\right) \text {. }
\end{aligned}
$$


Proof. i) $\left(H, \Omega_{1}\right) \widetilde{\widetilde{\subseteq}} 1_{\left(U_{I F H}, \Delta\right)}$, since $\theta_{H(\xi)}(u) \leq \theta_{H(\Delta)}(u)=1$ and

$\sigma_{H(\xi)}(u) \geq \sigma_{H(\Delta)}(u)=0$ for all $\xi \in \Delta, u \in U$,

ii) $0_{\left(U_{I F H}, \Delta\right)} \underset{\widetilde{\subseteq}}{ }\left(H, \Omega_{1}\right)$, since $0=\theta_{\tilde{\emptyset}}(u) \leq \theta_{H(\varepsilon)}(u)$ and $1=\sigma_{\tilde{\emptyset}}(u) \geq \sigma_{H(\xi)}(u)$ for all $\xi \in \Delta, u \in U$,

iii) $\left(H, \Omega_{1}\right) \widetilde{\subseteq}\left(G, \Omega_{2}\right) \Rightarrow \theta_{H(\xi)}(u) \leq \theta_{G(\xi)}(u)$ and $\sigma_{H(\xi)}(u) \geq \sigma_{H(\xi)}(u)$ for all $\xi \in$ $\Delta, u \in U$. Also $\left(G, \Omega_{2}\right) \widetilde{\simeq}\left(K, \Omega_{3}\right) \Rightarrow \theta_{G(\xi)}(u) \leq \theta_{K(\xi)}(u)$ and $\sigma_{G(\xi)}(u) \geq \sigma_{K(\xi)}(u)$ for all $\xi \in \Delta, u \in U$. Therefore $\theta_{H(\xi)}(u) \leq \theta_{K(\xi)}(u)$ and $\sigma_{H(\xi)}(u) \geq \sigma_{K(\xi)}(u)$. Thus, we obtain $\left(H, \Omega_{1}\right) \widetilde{\widetilde{\subseteq}}\left(K, \Omega_{3}\right)$.

Definition 14. The complement of intutionistic fuzzy hypersoft set $(H, \Omega)$ over the universe $U$ is denoted by $(H, \Omega)^{c}$ and defined as $(H, \Omega)^{c}=\left(H^{c}, \Omega\right)$, where $H^{c}:\left(E_{1} \times E_{2} \times \ldots \times E_{n}\right)=\Delta \rightarrow \operatorname{IFP}(U)$ and $H^{c}(\Omega)=(H(\Omega))^{c}$ for all $\Omega \subseteq \Delta$. Thus if $(H, \Omega)=\left\{\left\langle\xi,\left(\frac{u}{\theta_{H(\xi)}(u), \sigma_{H(\xi)}(u)}\right)>: u \in U, \xi \in \Omega\right)\right\}$, then $(H, \Omega)^{c}=\{<$ $\left.\left.\xi,\left(\frac{u}{\sigma_{H(\xi)}(u), \theta_{H(\xi)}(u)}\right)>: u \in U, \xi \in \Omega\right)\right\}$.

Example 15. According to Example 7 , consider the intuitionistic fuzzy hypersoft set $(H, \Omega)$ over the universe $U=\left\{\alpha_{1}, \alpha_{2}, \alpha_{3}, \alpha_{4}\right\}$.

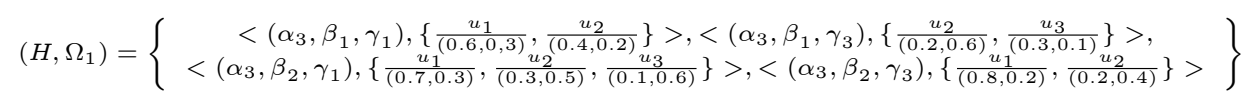

Then the complement of $(H, \Omega)$ is written as;

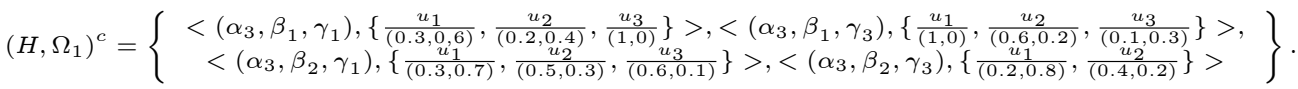

Theorem 16. Let $(H, \Omega)$ be any intuitionistic fuzzy hypersoft set over the universe U. Then,

i) $\left((H, \Omega)^{c}\right)^{c}=(H, \Omega)$

ii) $0_{\left(U_{I F H}, \Delta\right)}^{c}=1_{\left(U_{I F H}, \Delta\right)}$

iii) $1_{\left(U_{I F H}, \Delta\right)}^{c}=0_{\left(U_{I F H}, \Delta\right)}$

Proof. Proofs are trivial.

Definition 17. Let $U$ be an initial universe set, $\Omega_{1}, \Omega_{2} \subseteq \Delta$ and $\left(H, \Omega_{1}\right),\left(G, \Omega_{2}\right)$ be two intuitionistic fuzzy hypersoft sets over the universe $U$. The union of $\left(H, \Omega_{1}\right)$ and $\left(G, \Omega_{2}\right)$ is denoted by $\left(H, \Omega_{1}\right) \tilde{\cup}\left(G, \Omega_{2}\right)=\left(K, \Omega_{3}\right)$ where $\Omega_{3}=\Omega_{1} \cup \Omega_{2}$ and

$$
\begin{aligned}
\theta_{K(\xi)}(u) & = \begin{cases}H(\xi) & \text { if } \xi \in \Omega_{1}-\Omega_{2} \\
G(\xi) & \text { if } \xi \in \Omega_{2}-\Omega_{1} \\
\max (H(\xi),(G(\xi)) & \text { if } \xi \in \Omega_{1} \cap \Omega_{2}\end{cases} \\
\sigma_{K(\xi)}(u) & = \begin{cases}H(\xi) & \text { if } \xi \in \Omega_{1}-\Omega_{2} \\
G(\xi) & \text { if } \xi \in \Omega_{2}-\Omega_{1} \\
\min (H(\xi),(G(\xi)) & \text { if } \xi \in \Omega_{1} \cap \Omega_{2}\end{cases}
\end{aligned}
$$


Theorem 18. Let $U$ be an initial universe set, $\Omega_{1}, \Omega_{2}, \Omega_{3} \subseteq \Delta$ and $\left(H, \Omega_{1}\right),\left(G, \Omega_{2}\right)$, $\left(S, \Omega_{3}\right)$ be intuitionistic fuzzy hypersoft sets over the universe $U$. Then;

i) $\left(H, \Omega_{1}\right) \tilde{\cup}\left(H, \Omega_{1}\right)=\left(H, \Omega_{1}\right)$

ii) $0_{\left(U_{I F H}, \Delta\right)} \tilde{U}\left(H, \Omega_{1}\right)=\left(H, \Omega_{1}\right)$

iii) $\left(H, \Omega_{1}\right) \tilde{\cup} 1_{\left(U_{I F H}, \Delta\right)}=1_{\left(U_{I F H}, \Delta\right)}$

iv) $\left(H, \Omega_{1}\right) \widetilde{\cup}\left(G, \Omega_{2}\right)=\left(G, \Omega_{2}\right) \tilde{\cup}\left(H, \Omega_{1}\right)$

v) $\left(\left(H, \Omega_{1}\right) \tilde{\cup}\left(G, \Omega_{2}\right)\right) \tilde{\cup}\left(S, \Omega_{3}\right)=\left(H, \Omega_{1}\right) \tilde{\cup}\left(\left(G, \Omega_{2}\right) \tilde{\cup}\left(S, \Omega_{3}\right)\right)$

Proof. Proofs are trivial.

Definition 19. Let $U$ be an initial universe set, $\Omega_{1}, \Omega_{2} \subseteq \Delta$ and $\left(H, \Omega_{1}\right),\left(G, \Omega_{2}\right)$ be two intuitionistic fuzzy hypersoft sets over the universe $U$. The intersection of $\left(H, \Omega_{1}\right)$ and $\left(G, \Omega_{2}\right)$ is denoted by $\left(H, \Omega_{1}\right) \tilde{\cap}\left(G, \Omega_{2}\right)=\left(K, \Omega_{3}\right)$ where $\Omega_{3}=\Omega_{1} \cap \Omega_{2}$,

$\left.\left(K, \Omega_{3}\right)=\left\{<\xi,\left(\frac{u}{\left(\min \left\{\theta_{H(\xi)}(u), \theta_{G(\xi)}(u)\right\}, \max \left\{\theta_{H(\xi)}(u), \theta_{G(\xi)}(u)\right\}\right)}\right)>: u \in U, \xi \in \Omega\right)\right\}$

Theorem 20. Let $U$ be an initial universe set, $\Omega_{1}, \Omega_{2}, \Omega_{3} \subseteq \Delta$ and $\left(H, \Omega_{1}\right),\left(G, \Omega_{2}\right)$, $\left(S, \Omega_{3}\right)$ be intuitionistic fuzzy hypersoft sets over the universe $U$. Then;

i) $\left(H, \Omega_{1}\right) \tilde{\cap}\left(H, \Omega_{1}\right)=\left(H, \Omega_{1}\right)$

ii) $0_{\left(U_{I F H}, \Delta\right)} \tilde{\cap}\left(H, \Omega_{1}\right)=0_{\left(U_{I F H}, \Delta\right)}$

iii) $\left(H, \Omega_{1}\right) \tilde{\cap} 1_{\left(U_{I F H}, \Delta\right)}=\left(H, \Omega_{1}\right)$

iv) $\left(H, \Omega_{1}\right) \tilde{\cap}\left(G, \Omega_{2}\right)=\left(G, \Omega_{2}\right) \tilde{\cap}\left(H, \Omega_{1}\right)$

v) $\left(\left(H, \Omega_{1}\right) \tilde{\cap}\left(G, \Omega_{2}\right)\right) \tilde{\cap}\left(S, \Omega_{3}\right)=\left(H, \Omega_{1}\right) \tilde{\cap}\left(\left(G, \Omega_{2}\right) \tilde{\cap}\left(S, \Omega_{3}\right)\right)$

Proof. Proofs are trivial.

Definition 21. Let $U$ be an initial universe set, $\Omega_{1}, \Omega_{2} \subseteq \Delta$ and $\left(H, \Omega_{1}\right),\left(G, \Omega_{2}\right)$ be two intuitionistic fuzzy hypersoft sets over the universe $U$. The difference of $\left(H, \Omega_{1}\right)$ and $\left(G, \Omega_{2}\right)$ is denoted by $\left(H, \Omega_{1}\right) \widetilde{\backslash}\left(G, \Omega_{2}\right)=\left(K, \Omega_{3}\right)$ where

$\left(H, \Omega_{1}\right) \tilde{\cap}\left(G, \Omega_{2}\right)^{c}=\left(K, \Omega_{3}\right)$.

Example 22. We consider that attributes in Example 7 and Let $\left(H, \Omega_{1}\right),\left(G, \Omega_{2}\right)$ be any two intuitionistic fuzzy hypersoft set over the same universe $U=\left\{u_{1}, u_{2}, u_{3}\right\}$. The IFHSS $\left(H, \Omega_{1}\right)$ and $\left(G, \Omega_{2}\right)$ defined as follows;

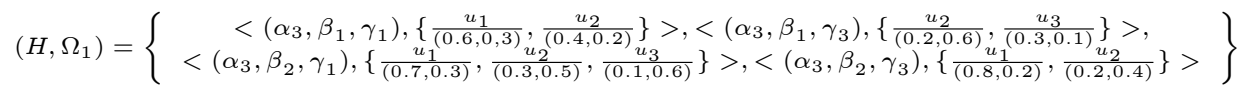

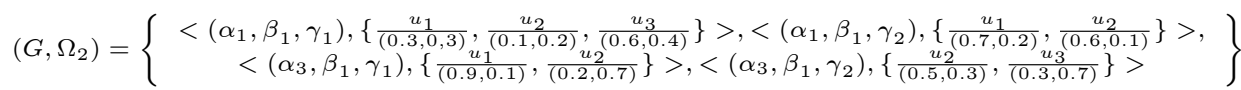

The union, intersection and difference operator of above IFHSS is written as;

$$
\left.\begin{array}{c}
\quad\left(H, \Omega_{1}\right) \tilde{\cup}\left(G, \Omega_{2}\right)= \\
<\left(\alpha_{3}, \beta_{1}, \gamma_{1}\right),\left\{\frac{u_{1}}{(0.9,0,1)}, \frac{u_{2}}{(0.4,0.2)}\right\}>,<\left(\alpha_{3}, \beta_{1}, \gamma_{3}\right),\left\{\frac{u_{2}}{(0.2,0.6)}, \frac{u_{3}}{(0.3,0.1)}\right\}>, \\
<\left(\alpha_{3}, \beta_{2}, \gamma_{1}\right),\left\{\frac{u_{1}}{(0.7,0.3)}, \frac{u_{2}}{(0.3,0.5)}, \frac{u_{3}}{(0.1,0.6)}\right\}>,<\left(\alpha_{3}, \beta_{2}, \gamma_{3}\right),\left\{\frac{u_{1}}{(0.8,0.2)}, \frac{u_{2}}{(0.2 .0 .4)}\right\}>, \\
<\left(\alpha_{1}, \beta_{1}, \gamma_{1}\right),\left\{\frac{u_{1}}{(0.3,0,3)}, \frac{u_{2}}{(0.1,0.2)}, \frac{u_{3}}{(0.6,0.4)}\right\}>,<\left(\alpha_{1}, \beta_{1}, \gamma_{2}\right),\left\{\frac{u_{1}}{(0.7,0.2)}, \frac{u_{2}}{(0.6,0.1)}\right\}> \\
<\left(\alpha_{3}, \beta_{1}, \gamma_{2}\right),\left\{\frac{u_{2}}{(0.5,0.3)}, \frac{u_{3}}{(0.3,0.7)}\right\}>
\end{array}\right\}
$$


and

$$
\left(H, \Omega_{1}\right) \tilde{\cap}\left(G, \Omega_{2}\right)=\left\{<\left(\alpha_{3}, \beta_{1}, \gamma_{1}\right),\left\{\frac{u_{1}}{(0.6,0,3)}, \frac{u_{2}}{(0.2,0.7)}\right\}>\right\}
$$

$$
\left(H, \Omega_{1}\right) \widetilde{\Upsilon}\left(G, \Omega_{2}\right)=\left\{<\left(\alpha_{3}, \beta_{1}, \gamma_{1}\right),\left\{\frac{u_{1}}{(0.1,0,9)}, \frac{u_{2}}{(0.4,0.2)}\right\}>\right\}
$$

Theorem 23. Let $U$ be an initial universe set, $\Omega_{1}, \Omega_{2} \subseteq \Delta$ and $\left(H, \Omega_{1}\right),\left(G, \Omega_{2}\right)$ be two intuitionistic fuzzy hypersoft sets over the universe $U$. Then De-Morgan Laws are hold.

i) $\left(\left(H, \Omega_{1}\right) \tilde{\cup}\left(G, \Omega_{2}\right)\right)^{c}=\left(H, \Omega_{1}\right)^{c} \tilde{\cap}\left(G, \Omega_{2}\right)^{c}$

ii) $\left(\left(H, \Omega_{1}\right) \tilde{\cap}\left(G, \Omega_{2}\right)\right)^{c}=\left(H, \Omega_{1}\right)^{c} \tilde{\cup}\left(G, \Omega_{2}\right)^{c}$

Proof. We only prove $\left(\left(H, \Omega_{1}\right) \tilde{\cup}\left(G, \Omega_{2}\right)\right)^{c}=\left(H, \Omega_{1}\right)^{c} \tilde{\cap}\left(G, \Omega_{2}\right)^{c}$. The other properties can be similarly proved. Suppose that $\left(\left(H, \Omega_{1}\right) \tilde{\cup}\left(G, \Omega_{2}\right)\right)^{c}=\left(K, \Omega_{1} \cup \Omega_{2}\right)$ and $\left(H, \Omega_{1}\right)^{c} \tilde{\cap}\left(G, \Omega_{2}\right)^{c}=\left(I, \Omega_{1} \cup \Omega_{2}\right)$. For any $\xi \in \Omega_{1} \cup \Omega_{2}$, we consider the following cases.

Case 1: $\xi \in \Omega_{1}-\Omega_{2}$. Then $K(\xi)=H^{c}(\xi)=I(\xi)$.

Case 2: $\xi \in \Omega_{2}-\Omega_{1}$. Then $K(\xi)=G^{c}(\xi)=I(\xi)$.

Case 3: $\xi \in \Omega_{1} \cap \Omega_{2}$. Then $K(\xi)=\left(\sigma_{H(\xi)}(u) \cap \sigma_{G(\xi)}(u), \theta_{H(\xi)}(u) \cup \theta_{G(\xi)}(u)\right)=$ $H^{c}(\xi) \cap G^{c}(\xi)=I(\xi)$.

Therefore, $K$ and $I$ are same operators, and so $\left(\left(H, \Omega_{1}\right) \tilde{\cup}\left(G, \Omega_{2}\right)\right)^{c}=\left(H, \Omega_{1}\right)^{c} \tilde{\cap}\left(G, \Omega_{2}\right)^{c}$.

Definition 24. Let $U$ be an initial universe set, $\Omega_{1}, \Omega_{2} \subseteq \Delta$ and $\left(H, \Omega_{1}\right),\left(G, \Omega_{2}\right)$ be two intuitionistic fuzzy hypersoft sets over the universe $U$. The "AND" operation on them is denoted by $\left(H, \Omega_{1}\right) \wedge\left(G, \Omega_{2}\right)=\left(K, \Omega_{1} \times \Omega_{2}\right)$ is given as;

$\left(K, \Omega_{1} \times \Omega_{2}\right)=\left\{<\left(\xi_{1}, \xi_{2}\right),\left(\frac{u}{\theta_{K\left(\xi_{1}, \xi_{2}\right)}(u), \sigma_{K\left(\xi_{1}, \xi_{2}\right)}(u)}\right)>: u \in U,\left(\xi_{1}, \xi_{2}\right) \in \Omega_{1} \times \Omega_{2}\right\}$

where

$$
\begin{aligned}
\theta_{K\left(\xi_{1}, \xi_{2}\right)}(u) & =\min \left\{\theta_{H\left(\xi_{1}\right)}(u), \theta_{G\left(\xi_{2}\right)}(u)\right\} \\
\sigma_{K\left(\xi_{1}, \xi_{2}\right)}(u) & =\max \left\{\sigma_{H\left(\xi_{1}\right)}(u), \sigma_{G\left(\xi_{2}\right)}(u)\right\}
\end{aligned}
$$

Definition 25. Let $U$ be an initial universe set, $\Omega_{1}, \Omega_{2} \subseteq \Delta$ and $\left(H, \Omega_{1}\right),\left(G, \Omega_{2}\right)$ be two intuitionistic fuzzy hypersoft sets over the universe $U$. The "OR" operation on them is denoted by $\left(H, \Omega_{1}\right) \vee\left(G, \Omega_{2}\right)=\left(K, \Omega_{1} \times \Omega_{2}\right)$ is given as;

$\left(K, \Omega_{1} \times \Omega_{2}\right)=\left\{<\left(\xi_{1}, \xi_{2}\right),\left(\frac{u}{\theta_{K\left(\xi_{1}, \xi_{2}\right)}(u), \sigma_{K\left(\xi_{1}, \xi_{2}\right)}(u)}\right)>: u \in U,\left(\xi_{1}, \xi_{2}\right) \in \Omega_{1} \times \Omega_{2}\right\}$

where

$$
\begin{aligned}
\theta_{K\left(\xi_{1}, \xi_{2}\right)}(u) & =\max \left\{\theta_{H\left(\xi_{1}\right)}(u), \theta_{G\left(\xi_{2}\right)}(u)\right\} \\
\sigma_{K\left(\xi_{1}, \xi_{2}\right)}(u) & =\min \left\{\sigma_{H\left(\xi_{1}\right)}(u), \sigma_{G\left(\xi_{2}\right)}(u)\right\}
\end{aligned}
$$


Theorem 26. Let $U$ be an initial universe set, $\Omega_{1}, \Omega_{2}, \Omega_{3} \subseteq \Delta$ and $\left(H, \Omega_{1}\right),\left(G, \Omega_{2}\right)$, $\left(S, \Omega_{3}\right)$ be intuitionistic fuzzy hypersoft sets over the universe $U$. Then;

i) $\left(H, \Omega_{1}\right) \vee\left[\left(G, \Omega_{2}\right) \vee\left(S, \Omega_{3}\right)\right]=\left[\left(H, \Omega_{1}\right) \vee\left(G, \Omega_{2}\right)\right] \vee\left(S, \Omega_{3}\right)$

ii) $\left(H, \Omega_{1}\right) \wedge\left[\left(G, \Omega_{2}\right) \wedge\left(S, \Omega_{3}\right)\right]=\left[\left(H, \Omega_{1}\right) \wedge\left(G, \Omega_{2}\right)\right] \wedge\left(S, \Omega_{3}\right)$

Proof. Straightforward.

Theorem 27. Let $U$ be an initial universe set, $\Omega_{1}, \Omega_{2} \subseteq \Delta$ and $\left(H, \Omega_{1}\right),\left(G, \Omega_{2}\right)$ be two intuitionistic fuzzy hypersoft sets over the universe $U$. Then;

i) $\left[\left(H, \Omega_{1}\right) \vee\left(G, \Omega_{2}\right)\right]^{c}=\left(H, \Omega_{1}\right)^{c} \wedge\left(G, \Omega_{2}\right)^{c}$

ii) $\left[\left(H, \Omega_{1}\right) \wedge\left(G, \Omega_{2}\right)\right]^{c}=\left(H, \Omega_{1}\right)^{c} \vee\left(G, \Omega_{2}\right)^{c}$.

Proof. We only prove (i). The other properties can be similarly proved.

For all $\left(\xi_{1}, \xi_{2}\right) \in \Omega_{1} \times \Omega_{2}$ and $u \in U$,

$$
\begin{aligned}
\left(H, \Omega_{1}\right) \vee\left(G, \Omega_{2}\right) & =\left\{<u, \max \left\{\theta_{H\left(\xi_{1}\right)}(u), \theta_{G\left(\xi_{2}\right)}(u)\right\}, \min \left\{\sigma_{H\left(\xi_{1}\right)}(u), \sigma_{G\left(\xi_{2}\right)}(u)\right\}>\right\}, \\
{\left[\left(H, \Omega_{1}\right) \vee\left(G, \Omega_{2}\right)\right]^{c} } & =\left\{<u, \min \left\{\sigma_{H\left(\xi_{1}\right)}(u), \sigma_{G\left(\xi_{2}\right)}(u)\right\}, \max \left\{\theta_{H\left(\xi_{1}\right)}(u), \theta_{G\left(\xi_{2}\right)}(u)\right\}>\right\}
\end{aligned}
$$

On the other hand,

$$
\begin{aligned}
& \left(H, \Omega_{1}\right)^{c}=\left\{<u, \sigma_{H\left(\xi_{1}\right)}(u), \theta_{H\left(\xi_{1}\right)}(u)>: \xi_{1} \in \Omega_{1}\right\} \\
& \left(G, \Omega_{2}\right)^{c}=\left\{<u, \sigma_{G\left(\xi_{2}\right)}(u), \theta_{G\left(\xi_{2}\right)}(u)>: \xi_{2} \in \Omega_{2}\right\}
\end{aligned}
$$

Then,

$$
\begin{aligned}
\left(H, \Omega_{1}\right)^{c} \wedge\left(G, \Omega_{2}\right)^{c} & =\left\{\left\{<u, \min \left\{\sigma_{H\left(\xi_{1}\right)}(u), \sigma_{G\left(\xi_{2}\right)}(u)\right\}, \max \left\{\theta_{H\left(\xi_{1}\right)}(u), \theta_{G\left(\xi_{2}\right)}(u)\right\}>\right\}\right\} \\
& =\left[\left(H, \Omega_{1}\right) \vee\left(G, \Omega_{2}\right)\right]^{c}
\end{aligned}
$$

Hence, $\left[\left(H, \Omega_{1}\right) \vee\left(G, \Omega_{2}\right)\right]^{c}=\left(H, \Omega_{1}\right)^{c} \wedge\left(G, \Omega_{2}\right)^{c}$ is obtained.

Example 28. We consider that attributes in Example 7 . Then the fuzzy hypersoft sets $\left(H, \Omega_{1}\right)$ and $\left(G, \Omega_{2}\right)$ defined as follows;

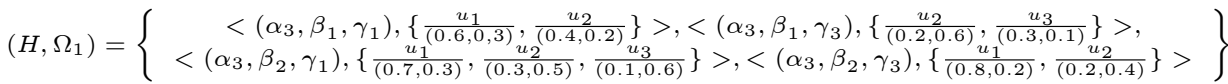

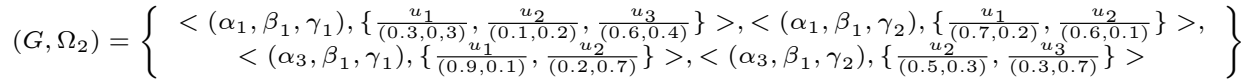

Let's assume $\left(\alpha_{3}, \beta_{1}, \gamma_{1}\right)=m_{1},\left(\alpha_{3}, \beta_{1}, \gamma_{3}\right)=m_{2},\left(\alpha_{3}, \beta_{2}, \gamma_{1}\right)=m_{3},\left(\alpha_{3}, \beta_{2}, \gamma_{3}\right)=$ $m_{4}$ in $\left(H, \Omega_{1}\right)$ and $\left(\alpha_{1}, \beta_{1}, \gamma_{1}\right)=n_{1},\left(\alpha_{1}, \beta_{1}, \gamma_{2}\right)=n_{2},\left(\alpha_{3}, \beta_{1}, \gamma_{1}\right)=n_{3},\left(\alpha_{3}, \beta_{1}, \gamma_{2}\right)=$ $n_{4}$ in $\left(G, \Omega_{2}\right)$ for easier operation. The tabular forms of these sets are as follows.

Then the "AND" and "OR" operations of these sets are given as below. 
TABLE 5. Tabular form of FHSS $\left(H, \Omega_{1}\right)$

\begin{tabular}{|cccc|}
\hline$\left(H, \Omega_{1}\right)$ & $u_{1}$ & $u_{2}$ & $u_{3}$ \\
$m_{1}$ & $(0.6,0,3)$ & $(0.4,0.2)$ & $(0,1)$ \\
$m_{2}$ & $(0,1)$ & $(0.2,0.6)$ & $(0.3,0.1)$ \\
$m_{3}$ & $(0.7,0.3)$ & $(0.3,0.5)$ & $(0.1,0.6)$ \\
$m_{4}$ & $(0.8,0.2)$ & $(0.2,0.4)$ & $(0,1)$ \\
\hline
\end{tabular}

TABle 6 . Tabular form of FHSS $\left(G, \Omega_{2}\right)$

\begin{tabular}{|cccc|}
\hline$\left(G, \Omega_{2}\right)$ & $u_{1}$ & $u_{2}$ & $u_{3}$ \\
$n_{1}$ & $(0.3,0,3)$ & $(0.1,0.2)$ & $(0.6,0.4)$ \\
$n_{2}$ & $(0.7,0.2)$ & $(0.6,0.1)$ & $(0,1)$ \\
$n_{3}$ & $(0.9,0.1)$ & $(0.2,0.7)$ & $(0,1)$ \\
$n_{4}$ & $(0,1)$ & $(0.5,0.3)$ & $(0.3,0.7)$ \\
\hline
\end{tabular}

TABLE 7. Tabular form of FHSS $\left(H, \Omega_{1}\right) \wedge\left(G, \Omega_{2}\right)$

\begin{tabular}{|cccc|}
\hline$\left(H, \Omega_{1}\right) \wedge\left(G, \Omega_{2}\right)$ & $u_{1}$ & $u_{2}$ & $u_{3}$ \\
$m_{1} \times n_{1}$ & $(0.3,0.3)$ & $(0.1,0.2)$ & $(0,1)$ \\
$m_{1} \times n_{2}$ & $(0.6,0.3)$ & $(0.4,0.2)$ & $(0,1)$ \\
$m_{1} \times n_{3}$ & $(0.6,0.3)$ & $(0.2,0.7)$ & $(0,1)$ \\
$m_{1} \times n_{4}$ & $(0,1)$ & $(0.4,0.3)$ & $(0,1)$ \\
$m_{2} \times n_{1}$ & $(0,1)$ & $(0.1,0.6)$ & $(0.3,0.4)$ \\
$m_{2} \times n_{2}$ & $(0,1)$ & $(0.2,0.6)$ & $(0,1)$ \\
$m_{2} \times n_{3}$ & $(0,1)$ & $(0.2,0.7)$ & $(0,1)$ \\
$m_{2} \times n_{4}$ & $(0,1)$ & $(0.2,0.6)$ & $(0.3,0.7)$ \\
$m_{3} \times n_{1}$ & $(0.3,0.3)$ & $(0.1,0.5)$ & $(0.1,0.6)$ \\
$m_{3} \times n_{2}$ & $(0.7,0.3)$ & $(0.3,0.5)$ & $(0,1)$ \\
$m_{3} \times n_{3}$ & $(0.7,0.3)$ & $(0.2,0.7)$ & $(0,1)$ \\
$m_{3} \times n_{4}$ & $(0,1)$ & $(0.3,0.5)$ & $(0.1,0.7)$ \\
$m_{4} \times n_{1}$ & $(0.3,0.3)$ & $(0.1,0.4)$ & $(0,1)$ \\
$m_{4} \times n_{2}$ & $(0.7,0.2)$ & $(0.2,0.4)$ & $(0,1)$ \\
$m_{4} \times n_{3}$ & $(0.8,0.2)$ & $(0.2,0.7)$ & $(0,1)$ \\
$m_{4} \times n_{4}$ & $(0,1)$ & $(0.2,0.4)$ & $(0,1)$ \\
\hline
\end{tabular}

\section{Conclusion}

The aim of this paper is to overcome the uncertainty trouble in more particular way by way of combing Intuitionistic fuzzy set with Hypersoft set. Some operations of Intuitionistic Fuzzy Hypersoft set such as subset, equal set, union, intersection, complement, AND, OR operations are presented. By defining these notions, the foundation of the intuitionistic fuzzy hypersoft set structure was built. 
TABLE 8. Tabular form of FHSS $\left(H, \Omega_{1}\right) \vee\left(G, \Omega_{2}\right)$

\begin{tabular}{|cccc|}
\hline$\left(H, \Omega_{1}\right) \vee\left(G, \Omega_{2}\right)$ & $u_{1}$ & $u_{2}$ & $u_{3}$ \\
$m_{1} \times n_{1}$ & $(0.6,0.3)$ & $(0.4,0.2)$ & $(0.6,0.4)$ \\
$m_{1} \times n_{2}$ & $(0.7,0.2)$ & $(0.6,0.1)$ & $(0,1)$ \\
$m_{1} \times n_{3}$ & $(0.9,0.1)$ & $(0.4,0.2)$ & $(0,1)$ \\
$m_{1} \times n_{4}$ & $(0.6,0.3)$ & $(0.5,0.2)$ & $(0.3,0.7)$ \\
$m_{2} \times n_{1}$ & $(0.3,0.3)$ & $(0.2,0.2)$ & $(0.6,0.1)$ \\
$m_{2} \times n_{2}$ & $(0.7,0.2)$ & $(0.6,0.1)$ & $(0.3,0.1)$ \\
$m_{2} \times n_{3}$ & $(0.9,0.1)$ & $(0.2,0.6)$ & $(0.3,0.1)$ \\
$m_{2} \times n_{4}$ & $(0,1)$ & $(0.5,0.3)$ & $(0.3,0.1)$ \\
$m_{3} \times n_{1}$ & $(0.7,0.3)$ & $(0.3,0.2)$ & $(0.6,0.4)$ \\
$m_{3} \times n_{2}$ & $(0.7,0.2)$ & $(0.6,0.1)$ & $(0.1,0.6)$ \\
$m_{3} \times n_{3}$ & $(0.9,0.1)$ & $(0.3,0.5)$ & $(0.1,0.6)$ \\
$m_{3} \times n_{4}$ & $(0.7,0.3)$ & $(0.5,0.3)$ & $(0.3,0.6)$ \\
$m_{4} \times n_{1}$ & $(0.8,0.2)$ & $(0.2,0.2)$ & $(0.6,0.4)$ \\
$m_{4} \times n_{2}$ & $(0.8,0.2)$ & $(0.6,0.1)$ & $(0,1)$ \\
$m_{4} \times n_{3}$ & $(0.9,0.1)$ & $(0.2,0.4)$ & $(0,1)$ \\
$m_{4} \times n_{4}$ & $(0.8,0.2)$ & $(0.5,0.3)$ & $(0.3,0.7)$ \\
\hline
\end{tabular}

The validity and implementation of the proposed operations and definitions are validated through presenting suitable instance. Matrices, similarity measure, single and multi-valued, interval valued, functions, distance measures, algorithms: score function, VIKOR, TOPSIS, AHP of Intutionistic Fuzzy Hypersoft sets will be future work. We hope that, this study will play a critical function in future decision-making research.

Authors Contribution Statement The authors contributed equally to this work. All authors of the submitted research paper have directly participated in the planning, execution, or analysis of study.

Declaration of Competing Interest The authors declare that there is no conflict of interest regarding the publication of this article.

\section{REFERENCES}

[1] Abbas, M., Murtaza, G., Smarandache, F., Basic operations on hypersoft sets and hypersoft point, Neutrosophic sets and system, 35 (2020).

[2] Aktaş, H., and Çağman, N., Soft sets and soft groups, Information sciences, 177(13) (2007), 2726-2735. https://doi.org/10.1016/j.ins.2006.12.008.

[3] Atanassov, K., Intuitionistic fuzzy sets, Fuzzy Sets and Systems, 20 (1986), 87-96.

[4] Atanassov, K., Intuitionistic Fuzzy Sets, Theory and Applications, PhysicaVerlag, Heidelberg, 1999. 
[5] Chen, D., Tsang, E., Yeung, D. S., and Wang, X., The parameterization reduction of soft sets and its applications, Computers \& Mathematics with Applications, 49(5-6) (2005), 757-763. https://doi.org/10.1016/j.camwa.2004.10.036

[6] Gayen, S., Smarandache, F., Jha, S., Singh, M. K., Broumi, S., \& Kumar, R. Introduction to plithogenic hypersoft subgroup. Neutrosophic Sets and Systems, 33(1) (2020), 14.

[7] Jiang, Y.,Tang, Y., Chen, Q., An adjustable approach to intuitionistic fuzzy soft sets based decision making, Applied Mathematical Modelling, 35 (2011), 824-836.https://doi.org/10. 1016/j.apm.2010.07.038

[8] Khameneh, A. Z., \& Kılı̧̧man, A. Multi-attribute decision-making based on soft set theory: A systematic review. Soft Computing, 23(16) (2019), 6899-6920. https://doi.org/10.1007/ s00500-018-3330-7

[9] Kong, Z., Gao, L., Wang, L., and Li, S., The normal parameter reduction of soft sets and its algorithm. Computers 8 Mathematics with Applications, 56(12) (2008), 3029-3037 https: //doi.org/10.1016/j.camwa.2008.07.013

[10] Maji, P. K., More on intuitionistic fuzzy soft sets, in: H. Sakai, M.K. Chakraborty, A.E. Hassanien, D. Slezak, W. Zhu (Eds.), Proceedings of the 12th International Conference on Rough Sets, Fuzzy Sets, Data Mining and Granular Computing (RSFDGrC 2009), Lecture Notes in Computer Science, 5908, Springer, 2009, 231-240.

[11] Maji, P. K., An application of intuitionistic fuzzy soft sets in a decision making problem, IEEE International Conference on Progress in Informatics and Computing (PIC), 10-12 (2010), 349-351.

[12] Maji, P. K., Biswas, R., and Roy, A. Soft set theory. Computers 83 Mathematics with Applications, 45(4-5) (2003), 555-562. https://doi.org/10.1016/S0898-1221(03)00016-6

[13] Maji, P. K., Biswas, R., and Roy, A., Intuitionistic fuzzy soft sets, Journal of Fuzzy Mathematics, 9(3) (2001), 677-692.

[14] Maji, P., Roy, A. R., and Biswas, R. An application of soft sets in a decision making problem, Computers 83 Mathematics with Applications, 44(8-9) (2002), 1077-1083. https://doi.org/ 10.1016/S0898-1221(02) 00216-X

[15] Molodtsov, D., Soft set theory first results, Computers $\&$ Mathematics with Applications, 37(4-5) (1999), 19-31. https://doi.org/10.1016/S0898-1221(99)00056-5

[16] Ozturk, T. Y., Bayramov, S., Soft mappings space. The Scientific World Journal, 2014 (2014). https://doi.org/10.1155/2014/307292

[17] Ozturk, T. Y., Aras, C. G., Bayramov, S., A new approach to operations on neutrosophic soft sets and to neutrosophic soft topological spaces. Communications in Mathematics and Applications, 10(3) (2019), 481-493.

[18] Ozturk, T. Y., Yolcu, A., On Neutrosophic Hypersoft Topological Spaces, Theory and Application of Hypersoft Set, Pons Publishing House, Brussel, 2021, 215-234.

[19] Pei, D., and Miao, D., From soft sets to information systems. IEEE International Conference on Granular Computing, (2005), 617-621.

[20] Saeed, M., Ahsan, M., Siddique, M. K., Ahmad, M. R., A Study of the fundamentals of hypersoft set theory, International Journal of Scientific \&5 Engineering Research, 11(1) (2020), $320-329$.

[21] Smarandache, F., Extension of soft set to hypersoft set, and then to plithogenic hypersoft set, Neutrosophic sets and system. 22, (2018), 168-170.

[22] Saqlain M, Saeed M, Ahmad M. R, Smarandache F, Generalization of TOPSIS for neutrosophic hypersoft set using accuracy function and its application, Neutrosophic Sets and Systems, 27 (2019), 131-137.

[23] Saqlain M, Sana M, Jafar N, Saeed. M, Said. B. Single and multi-valued neutrosophic hypersoft set and tangent similarity measure of single valued neutrosophic hypersoft sets, Neutrosophic Sets and Systems, 32, (2020). 
[24] Saqlain, M., Moin, S., Jafar, M. N., Saeed, M., \& Smarandache, F., Aggregate Operators of neutrosophic hypersoft set. Neutrosophic Sets and Systems, 32(1) (2020), 18.

[25] Yolcu, A., Ozturk, T. Y., Fuzzy Hypersoft Sets and It's Application to Decision-Making, Theory and Application of Hypersoft Set, Pons Publishing House, Brussel, 2021, 50-64.

[26] Zadeh, L. A., Fuzzy sets. Information and control, 8(3) (1965), 338-353.

[27] Zhang, X., Park, C., Wu, S., Soft set theoretical approach to pseudo-BCI algebras, Journal of Intelligent \& Fuzzy Systems, 34(1) (2018), 559-568. https://doi.org/10.3233/JIFS-17777

[28] Zou, Y., and Xiao, Z., Data analysis approaches of soft sets under incomplete information. Knowledge-Based Systems 21(8) (2008), 941-945. 